\title{
TRANSMISSION OF HEATER GENERATED HIGH FREQUENCY PHONONS THROUGH A SAPPHIRE-He II BOUNDARY
}

\author{
J. BUCK, K. LASSMANN and W. EISENMENGER \\ Physikalisches Institut der Universität Stuttgart, Teilinstitut 1, Germany
}

Received 29 October 1974

\begin{abstract}
We report on a new method to measure the absolute transmission coefficient through a solid-He II-boundary by determining absolutely the temperature amplitude of the second sound pulses in the liquid.
\end{abstract}

Recently, Swanenburg and Wolter [1] demonstrated that the measurement of second sound excited by phonons incident on a solid-He II boundary is a useful method to obtain information on the phonon transmission. In their experiment the ratio of the longitudinal and transverse wave transmission coefficients could be determined. We report on a similar experiment by which the absolute value of the total transmission coefficient is obtained from the temperature amplitude of the second sound pulse using a superconducting Sn-I-Sn tunneling junction as a fast thermometer calibrated on the basis of the nonlinear propagation properties of second sound. In our experiment a constantan heater $\left(0.678 \mathrm{~mm}^{2}\right)$ was evaporated on one end of a sapphire crystal (mechanically polished, $1 \mathrm{~cm}$ diameter, $1 \mathrm{~cm}$ thick). Phonons generated by applying voltage pulses to the heater and incident on the opposite end of the crystal are partly transmitted and excite second sound. The superconducting Sn-tunnel junction, $1 \mathrm{~mm}$ apart, $1 \mathrm{~mm}^{2}$, evaporated on glass, serves as a detector for the second sound. Contrary to [1] separate observation of second sound pulses caused by longitudinal and transverse phonons was not possible with our setup due to mean free path effects in the He II (see [1]).

In a preparatory step we show, that there is a simple relation between second sound temperature amplitude and detector signal.

The signal current of the junction biased below $2 \Delta_{\mathrm{Sn}} / e$ is given by (small signal limit)

$J_{\mathrm{s}}=\frac{\partial J_{\mathrm{th}}}{\partial T} T_{\mathrm{D}}$

( $J_{\text {th }}=$ thermal tunneling current, $T_{\mathrm{D}}=$ deviation of the quasiparticle occupation temperature from equilibrium).

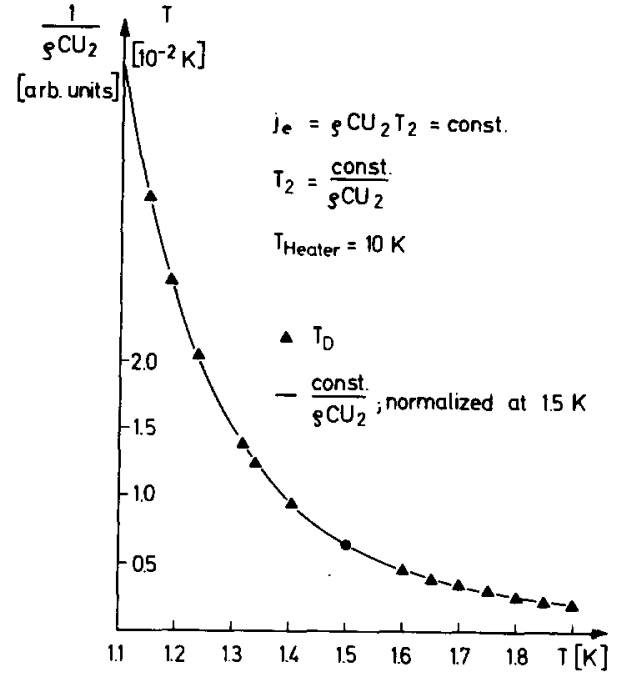

Fig. 1. Comparison of $T_{\mathrm{D}}$ and $T_{2}$

Making use of the relation

$j_{\mathrm{e}}=\rho C u_{2} T_{2}$

$\left(j_{\mathrm{e}}=\right.$ energy current density in second sound, $\rho . C=$ specific heat per volume, $u_{2}=$ velocity of second sound) and holding $j_{\mathrm{e}}=$ const experimentally provides a second sound temperature amplitude $T_{2}=$ const/ $\rho \mathrm{Cu}_{2}$ known to a constant factor in its variation with bath temperature. The measurements show that $T_{\mathrm{D}} \propto 1 / \rho C u_{2} \propto T_{2}$ (fig. 1).

The constant of proportionality relating $T_{\mathrm{D}}$ and $T_{2}$ is obtained by making use of the amplitude dependence of the velocity of second sound $[2,3]$. The velocity of second sound of finite amplitude with no shock front developed is $u_{2}=u_{20}+\alpha_{2} v_{n}$, where $u_{20}$ 


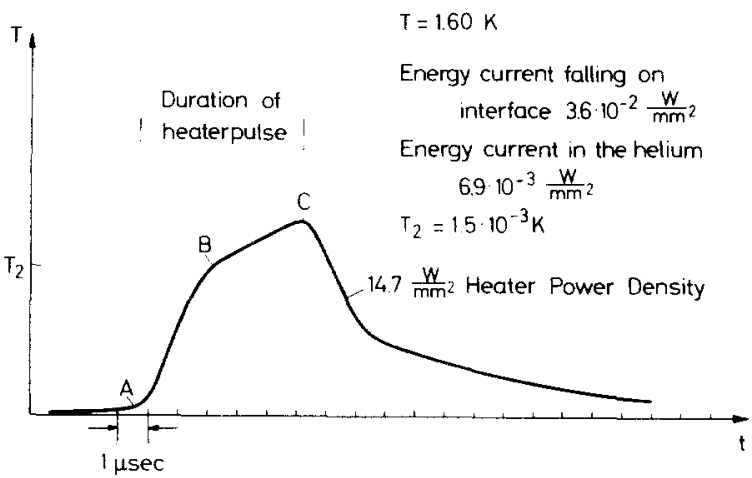

Fig. 2. Typical second sound pulse signal as detected by the tunnel junction.

is the velocity for vanishing amplitude and $v_{n}$ the velocity of the normal fluid. $\alpha_{2}$ has been determined experimentally [3]. Using $T_{2}=\left(S T / \mathrm{Cu}_{2}\right) v_{n}$ we obtain

$u_{2}=u_{20} /\left(1-\alpha_{2} C T_{2} / S T\right)$

$S=$ entropy, $T=$ bath temperature.

From the last formula it is seen, that $T_{2}$ can be measured by time of flight experiments with pulses of finite amplitude.

The calibration was performed by observing the maximum of the pulse. We find $T_{\mathrm{D}}=1.35 T_{2}$ in accordance with an estimate taking account of the reflection of second sound at the junction of the heat current in to the substrate.

The transmission coefficient of the sapphire-helium boundary is given by the ratio of the energy current density in the helium, as follows from eq. (2) with $T_{2}$ and in the sapphire. The energy current in the crystal was calculated from formulae given by Weis [4] valid for sufficiently high heater powers leading to the formation of a bubble of helium gas at the heater-helium interface effectively reducing direct phonon emission into the helium bath. A pulse signal typical for the second sound pulses detected is shown in fig. 2. The signal amplitude $A B$ is due to phonons travelling directly from the heater to the opposite side of the crystal, whereas the additional rise (BC) is due to phonons having undergone multiple reflections in the crystal. Thus, $T_{2}$ at point $\mathrm{B}$ is related to the energy flux in the crystal calculated in the beforementioned way. The transmission coefficient we find is $21 \%$ for heater temperature amplitudes from 6 to $20 \mathrm{~K}$ corresponding to phonon frequencies of $400 \mathrm{GHz}$ to $1.2 \mathrm{THz}$. In our experiment this is an average over the longitudinal and transverse polarizations. It is known, however, that the transmission coefficients of longitudinal and transverse phonons into liquid He do not differ very much $[1,6]$. Our value is in agreement with what Guo and Maris [6] find from a reflection experiment.

Corrections [5] made for neglecting the direct en. ergy flux from the heater into the helium bath shift the calculated transmission coefficient at $400 \mathrm{GHz}$ to about $38 \%$. Ishiguro and Fjeldly [5] find that the isolation properties of the gas bubble increase with increasing heater temperature. Taking their data the calculated transmission coefficients would decrease with increasing heater temperature, (or average frequency) in accordance with [7]

\section{References}

11] T.J.B. Swanenburg and J. Wolter, Phys, Rev, Lett. 31 (1973) 693.

[2] I.M. Chalatnikov, lortschritte der Physik 5 (1957) 287.

[3] A.J. Dessler and W.M. Tairbank, Phys. Rev. 104 (1956) 6.

[4] O. Weis, Z. Angew. Phys. 26 (1969) 325.

[5] T. Ishiguro and T.A. Fjeldly, Phys. Lett. 45A (1973) 127.

[6] C.J. Guo and H.J. Maris, Phys. Rev. Lett. 29 (1972) 855.

17] J. Wolter and T.J.B. Swanenburg, talk given at the 1974 Conf. of the Deutsche Physikalische Gesellschaft at Freudenstadt 